\title{
Double cocktail immunostains with high molecular weight cytokeratin and GATA-3: useful stain to discriminate in situ involvement of prostatic ducts or acini from stromal invasion by urothelial carcinoma in the prostate
}

\author{
Junghye Lee ${ }^{1}$, Youngeun Yoo ${ }^{1}$, Sanghui Park ${ }^{1}$, Min-Sun Cho ${ }^{1}$, Sun Hee Sung ${ }^{1}$, Jae Y. Ro ${ }^{2}$ \\ 'Department of Pathology, Ewha Womans University College of Medicine, Seoul, Korea; \\ 2Department of Pathology and Genomic Medicine, Houston Methodist Hospital and Weill Medical College of Cornell University, Houston, TX, USA
}

\begin{abstract}
Background: Distinguishing prostatic stromal invasion (PSI) by urothelial carcinoma (UC) from in situ UC involving prostatic ducts or acini with no stromal invasion (in situ involvement) may be challenging on hematoxylin and eosin stained sections. However, the distinction between them is important because cases with PSI show worse prognosis. This study was performed to assess the utility of double cocktail immunostains with high molecular weight cytokeratin (HMWCK) and GATA-3 to discriminate PSI by UC from in situ UC involvement of prostatic ducts or acini in the prostate. Methods: Among 117 radical cystoprostatectomy specimens for bladder UCs, 25 cases showed secondary involvement of bladder UC in prostatic ducts/acini only or associated stromal invasion and of these 25 cases, seven cases revealed equivocal PSI. In these seven cases with equivocal PSI, HMWCK, and GATA-3 double immunohistochemical stains were performed to identify whether this cocktail stain is useful to identify the stromal invasion. Results: In all cases, basal cells of prostate glands showed strong cytoplasmic staining for HMWCK and UC cells showed strong nuclear staining for GATA-3. In cases with stromal invasion of UC, GATA-3-positive tumor cells in the prostatic stroma without surrounding HMWCK-positive basal cells were highlighted and easily recognized. Among seven equivocal cases, two cases showed PSI and five in situ UC in the prostate. In two cases, the original diagnoses were revised. Conclusions: Our study suggested that HMWCK and GATA-3 double stains could be utilized as an adjunct method in the distinction between PSI by UC from in situ UC involving prostatic ducts or acini.
\end{abstract}

Key Words: Urothelial carcinoma; Prostatic stromal invasion; High molecular weight cytokeratin; GATA-3

Received: July 31, 2019 Revised: October 18, 2019 Accepted: November 12, 2019

Corresponding Author: Sanghui Park, MD, PhD, Department of Pathology, Ewha Womans University College of Medicine, 1071 Anyangcheon-ro, Yangcheon-gu, Seoul 07985, Korea

Tel: +82-2-2650-5044, Fax: +82-2-2650-2879, E-mail: spark0430@ewha.ac.kr

Corresponding Author: Min-Sun Cho, MD, PhD, Department of Pathology, Ewha Womans University College of Medicine, 25 Magokdong-ro 2-gil, Gangseo-gu, Seoul 07084, Korea

Tel: +82-2-6986-3422, Fax: +82-2-6986-7013, E-mail: mcho1124@ewha.ac.kr

Primary urothelial carcinoma (UC) of the prostate is rare, representing only about $1 \%$ to $4 \%$ of all prostate malignancies [1-3]. In contrast, secondary involvement of primary bladder UC is relatively common with an incidence of $12 \%$ in an early report [4]. However, the true incidence of prostatic involvement is more common. In several studies using whole-mount prostate sectioning technique, the incidence of prostatic involvement has been reported up to $48 \%$ (average, 35.5\%) [5-9]. The incidence of prostatic stromal invasion (PSI) ranges from $7.6 \%$ to $16.6 \%$ [4,6,9-13]. In 7th American Joint Committee on Cancer (AJCC) staging system, primary bladder UCs with PSI are classified as pT4a and correlated with worse prognosis [14]. However, the staging of continuous subepithelial PSI from in situ carcinoma was not designated properly. The 8th AJCC staging system clarified the staging of PSI according to the mode of invasion: primary bladder UCs with direct PSI via transmural route is designated as T4a while continuous subepithelial PSI from in situ carcinoma is now classified as T2 [15]. Although the presence of PSI does not affect the current 8th AJCC staging of muscleinvasive primary bladder UCs $(\geq \mathrm{T} 2)$, it is still important to distinguish PSI from in situ UC because PSI is associated with poor prognosis [16]. Therefore, the correct identification of PSI with primary bladder UC is of paramount importance for accurate staging and predicting patients' prognoses. However, the 
distinction between PSI by UCs from only in situ UC involvement of prostatic ducts or acini in the prostate can be challenging on hematoxylin and eosin $(\mathrm{H} \& \mathrm{E})$ stained slides examination alone in some cases.

In this study, double cocktail immunostains with high molecular weight cytokeratin (HMWCK) and GATA-3 were performed to determine its usefulness for detecting PSI.

\section{MATERIALS AND METHODS}

\section{Cases selection}

Surgical pathology files of our institute from 2000 to 2012 were searched for radical cystectomy cases of prostatic involvement by primary bladder UCs with available tissue blocks. Among 164 radical cystectomy cases for primary bladder UCs, 140 were males and concomitant prostatectomy was performed in 117 cases (117/140, 83.6\%). Among 117 radical cystoprosta- tectomy specimens for bladder UCs, 25 cases (21.4\%) showed secondary involvement of bladder UC in prostatic ducts/acini only or associated stromal invasion, and of these 25 cases, seven cases revealed equivocal PSI. The remaining 18 cases revealed unequivocal prostatic involvement with either in situ only or PSI. PSI was considered equivocal at $\mathrm{H} \& \mathrm{E}$ level when one of the following features was present: (1) in situ UC in prostatic ducts with irregular and blurred outlines and a basal layer that was not well defined (pattern 1) and (2) in situ UC in prostatic ducts surrounded by desmoplastic stroma with or without inflammatory infiltrate (pattern 2). Slides were re-reviewed independently by two experienced pathologists (S.P. and M.S.C.). They had concordant interpretation in 18 cases and had discordant interpretation in seven cases due to equivocal stromal invasion.

\section{Double immunohistochemistry}

Double immunohistochemical stains were performed with
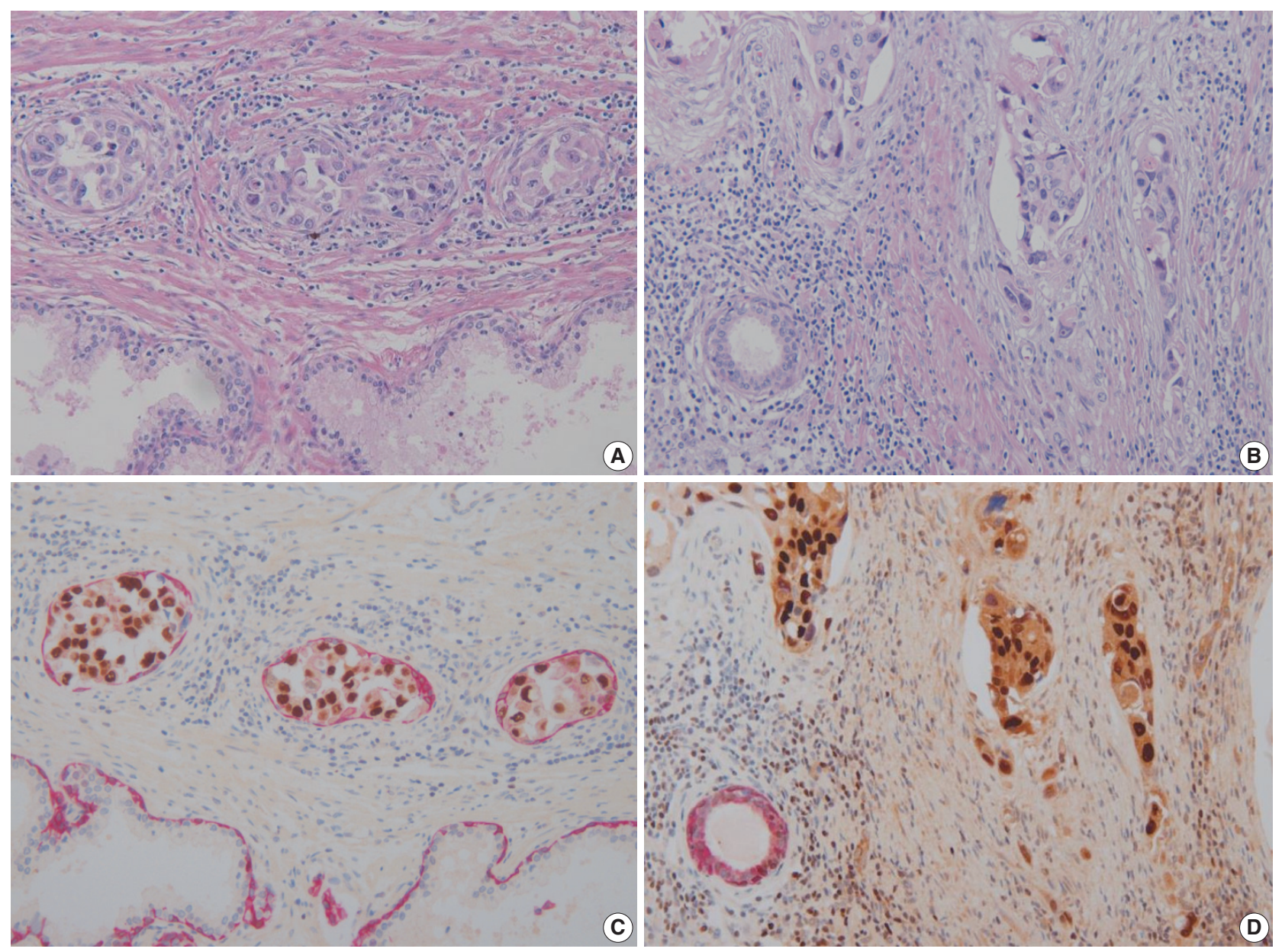

Fig. 1. (A) Urothelial carcinoma in situ involving prostatic ducts. (B) Urothelial carcinoma with prostatic stromal invasion. (C) Urothelial carcinoma in situ involving prostatic ducts surrounded by basal cells (double immunohistochemical stain). (D) Urothelial carcinoma with prostatic stromal invasion without surrounding basal cells (double immunohistochemical stain). 
mouse monoclonal antibodies against human HMWCK (1:200 dilution, $34 \beta E 12$ clone, Novocastra, Newcastle upon Tyne, UK) and GATA-3 (1:100 dilution, L50-823 clone, Cell Marque, Rocklin, CA, USA) using formalin-fixed paraffin-embedded tissue blocks of tumors with adjacent benign prostatic tissue. Four- $\mu \mathrm{m}$ sections were dried at $55^{\circ} \mathrm{C}$ for 3 hours, then subjected to heat-induced epitope retrieval, 30 minutes for HMWCK and 20 minutes for GATA-3, at pH 9.0 on a Leica-Bond Autostainer (Leica Biosystems, Newcastle upon Tyne, UK). Bond Polymer Refine Detection Kit was used and the sections were counterstained with hematoxylin. Subsequently, double-stain combinations using HMWCK and GATA-3 were tested in some cases using different combinations of brown and red chromogens. Bond Polymer Refine Brown Detection Kit (Leica Biosystems) was used for detection of GATA-3 and Bond Polymer Refine Red Detection Kit for detection of HMWCK. All cases contained foci of $\mathrm{UC}$ with colonization of prostatic ducts and acini that were used as GATA-3 positive control. Adjacent benign prostatic glands were used as an internal control for HMWCK stain (Fig. 1). Pancytokeratin stain (1:200 dilution, AE1/AE3, Novocastra) was performed for case 6.

\section{Ethics statement}

All procedures performed in the current study were approved by the international review board at Ewha Womans University Hospital (2016-09-020) in accordance with the 1964 Helsinki declaration and its later amendments. Formal written informed consent was not required with a waiver by the IRB.

\section{RESULTS}

Clinicopathological data on these seven patients with equivocal PSI are summarized in Table 1. The patients' mean age was of 73.3 years at diagnosis (range, 60 to 93 years). Among seven cases, six showed conventional muscle invasive high-grade UC and one showed diffuse and multifocal UC in situ throughout the bladder (case 1). Six showed conventional high-grade UC morphology, but one case (case 4) showed several different histologic features (squamous, glandular and neuroendocrine differentiations). Lymphovascular invasion was identified in three cases. Five cases were free of tumor at margins and two showed positive margins, one in left ureter and the other in the urethra.

In these seven cases, UC involvement was seen in prostatic ducts and acini with equivocal PSI. Four cases revealed pattern 1 equivocal invasion (Fig. 2A) and three cases pattern 2 equivocal invasion (Figs. 2B, 3A, B). Originally, two cases, both with pattern 2 equivocal invasion, were diagnosed as having PSI and the remaining five cases, four pattern 1 and one pattern 2 cases, with equivocal invasion were diagnosed as no PSI.

In these seven cases, double cocktail immunostains were performed. Prostatic basal cells surrounding in situ UC stained positively for HMWCK and UC cells stained for GATA-3 (Fig. 1A, C). In all cases, UC cells stained positively for GATA-3 (7/7, $100 \%$ ). The combination of HMWCK (red)/GATA-3 (brown) easily distinguish in situ from invasive tumor (Fig. 1B, D). Among seven equivocal cases, two cases showed PSI and five cases in situ UC in the prostate. Based on these stain results, cases 5 and 6 were revised according to the 7 th AJCC staging system and

Table 1. Clinicopathologic features in patients with equivocal prostatic stromal invasion

\begin{tabular}{|c|c|c|c|c|c|c|c|c|c|c|c|c|}
\hline $\begin{array}{l}\text { Case } \\
\text { No. }\end{array}$ & Age/Sex & $\begin{array}{l}\text { Histology } \\
\text { (variant) }\end{array}$ & LVI & $\begin{array}{l}\text { Margin } \\
\text { status }\end{array}$ & $\begin{array}{l}\text { Equivocal } \\
\text { pattern }\end{array}$ & $\begin{array}{c}\text { PSI, } \\
\text { original } \\
\text { (H\&E) }\end{array}$ & $\begin{array}{c}\mathrm{PSI}, \\
\text { revised } \\
(\mathrm{IHC})\end{array}$ & $\begin{array}{l}\text { AJCC 7th } \\
\mathrm{T} \text { (original) }\end{array}$ & $\begin{array}{l}\text { AJCC 7th } \\
\mathrm{T} \text { (revised) }\end{array}$ & $\begin{array}{c}\text { AJCC 8th } \\
T\end{array}$ & Treatment & $\mathrm{F} / \mathrm{U}(\mathrm{mo})$ \\
\hline 1 & $\mathrm{M} / 71$ & UC in situ (C) & Absent & Negative & 1 & No & No & Tis & Tis & Tis & TURBT, RCP & LTF \\
\hline 2 & M/61 & UC, HG (C) & Absent & Negative & 1 & No & No & $\mathrm{T} 1$ & $\mathrm{~T} 1$ & $\mathrm{~T} 1$ & $\begin{array}{l}\text { TURBT, BCG, } \\
\text { neoadjuvant CTx, RCP }\end{array}$ & AWD (56) \\
\hline 3 & M/93 & UC, HG (C) & Absent & Negative & 1 & No & No & T3 & T3 & T3 & TURBT, RCP & DOD (10) \\
\hline 4 & M/79 & $\begin{array}{l}\mathrm{UC}, \mathrm{HG} \\
\qquad(\mathrm{S}, \mathrm{G}, \mathrm{N})\end{array}$ & Present & Negative & 1 & No & No & T3 & T3 & T3 & $\begin{array}{l}\text { TURBT, neoadjuvant CTx, } \\
\text { RCP, adjuvant CTx }\end{array}$ & DOD (2) \\
\hline 5 & $\mathrm{M} / 60$ & UC, HG (C) & Absent & $\begin{array}{l}\text { Positive, } \\
\text { left ureter }\end{array}$ & 2 & Yes & No & $\mathrm{T} 4 \mathrm{a}$ & $\mathrm{T} 1$ & $\mathrm{~T} 1$ & TURBT, BCG, RCP & AWD (54) \\
\hline 6 & M/85 & UC, HG (C) & Present & Negative & 2 & No & Yes & T3 & $\mathrm{T} 4 \mathrm{a}$ & T3 & $\begin{array}{l}\text { TURBT, RCP, } \\
\text { adjuvant CTx }\end{array}$ & DOD (16) \\
\hline 7 & $M / 64$ & UC, HG (C) & Present & $\begin{array}{l}\text { Positive, } \\
\text { urethra }\end{array}$ & 2 & Yes & Yes & $\mathrm{T} 4 \mathrm{a}$ & $\mathrm{T} 4 \mathrm{a}$ & $\mathrm{T} 2$ & $\begin{array}{c}\text { TURBT, BCG, neoadjuvant } \\
\text { CTx, RCP, adjuvant CTx }\end{array}$ & AWD (58) \\
\hline
\end{tabular}

LVI, Iymphovascular invasion; PSI, prostatic stromal invasion; H\&E, hematoxylin and eosin; IHC, immunohistochemistry; AJCC, American Joint Committee on Cancer; F/U, follow-up; M, male; UC, urothelial carcinoma; C, conventional; TURBT, transurethral resection of bladder tumor; RCP, radical cystoprostatectomy; LTF, loss to follow up; HG, high grade; BCG, bacillus Calmette-Guerin; CTx, chemotherapy; AWD, alive with disease; DOD, died of disease; S, squamous differentiation; $\mathrm{G}$, glandular differentiation; N, neuroendocrine differentiation. 

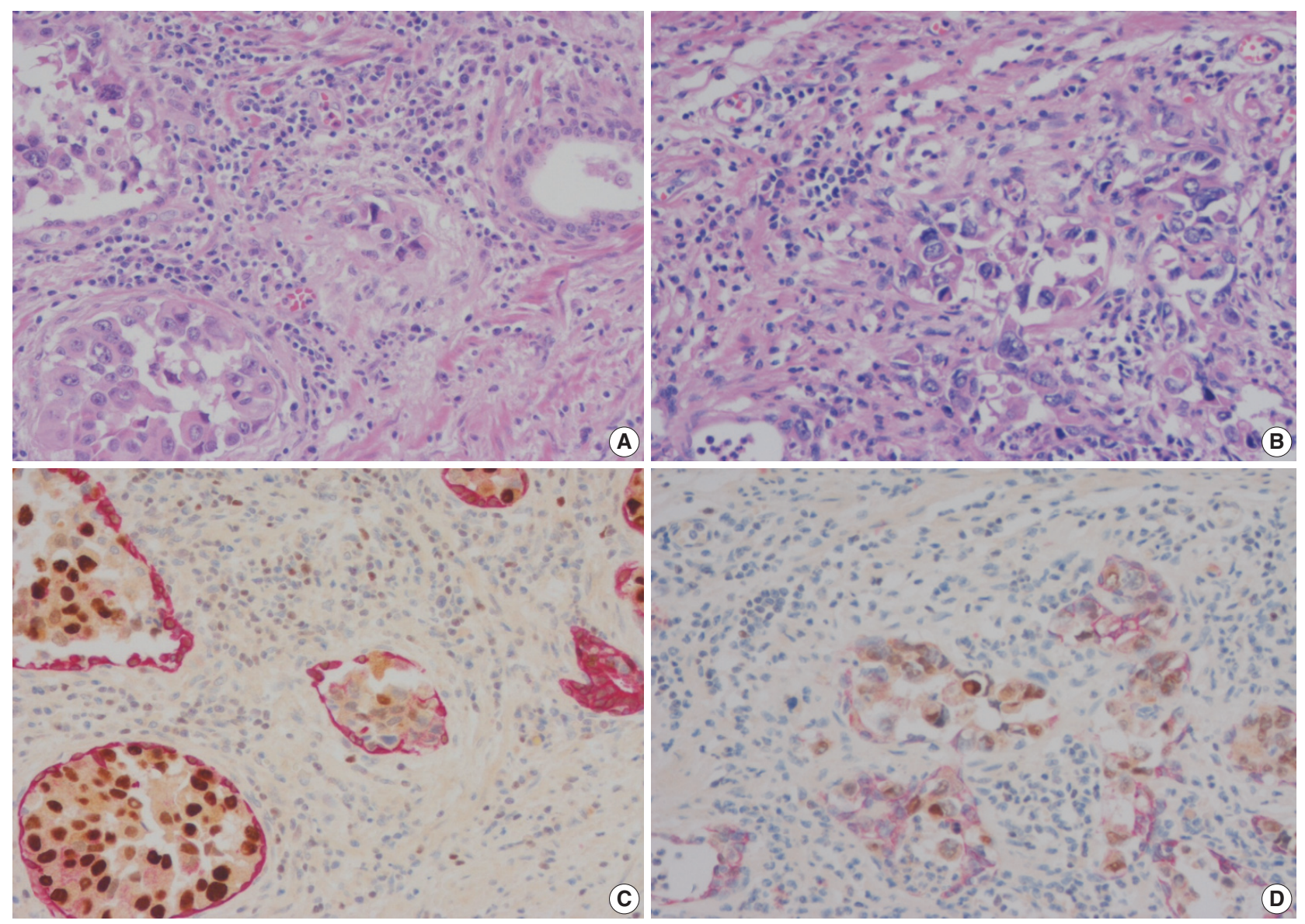

Fig. 2. (A) Urothelial carcinoma in situ with equivocal stromal invasion (equivocal pattern 1). (B) Urothelial carcinoma in situ with equivocal stromal invasion (equivocal pattern 2). (C) Urothelial carcinoma in situ with intact surrounding basal cells (double immunohistochemical stain). (D) Urothelial carcinoma in situ with disrupted but sustained basal cells (double immunohistochemical stain).

cases 5 and 7 were revised according to the 8th AJCC system. In one case, the original T4a classification was revised as $\mathrm{T} 1$ and in the other case, the original T3 classification was revised to T4a according to the 7th AJCC staging system. According to the 8th AJCC staging system, one case classified originally as T4a was revised as T1, and the other T4a case was revised as T2.

All patients received radical cystoprostatectomy after transurethral resection of bladder tumor and additional treatments such as bacillus Calmette-Guerin (BCG) instillation, neoadjuvant chemotherapy and adjuvant chemotherapy were given as needed. Three patients (cases 1, 7, and 5) are alive with disease at 21, 26, and 54 months, respectively. Other three patients (cases 4, 3, and 6) died of disease at 2-, 10-, and 16-month follow-up, respectively, and the remaining one patient (case 2) was lost to follow up.

In equivocal cases with pattern 1, GATA-3-positive tumor cells in prostatic ducts/acini were surrounded by HMWCKpositive basal cells (Fig. 2C). In three equivocal cases with pat- tern 2, two cases were revised after performing double immunohistochemistry (IHC). In one case, double IHC revealed the presence of preserved basal cells with continuous, linear and focally weak staining pattern of HMWCK at the periphery of the involved prostatic ducts/acini (case 5) (Fig. 2D). This case, originally signed out as PSI, was revised as in situ UC involving prostatic ducts/acini without PSI. In the other case (case 6), intense aggregates of inflammatory cells were identified adjacent to prostatic ducts (Fig. 3A, B). GATA-3-positive and HMWCKnegative singly scattered invasive tumor cells were present within the periductal inflammatory area (Fig. 3C). Singly scattered tumor cells were also highlighted by pancytokeratin stain, supporting invasive UC cells (Fig. 3D). This case, originally signed out as in situ UC involving prostatic ducts, was revised as having PSI.

\section{DISCUSSION}

It is well-known that UCs with extensive PSI has a poor prog- 

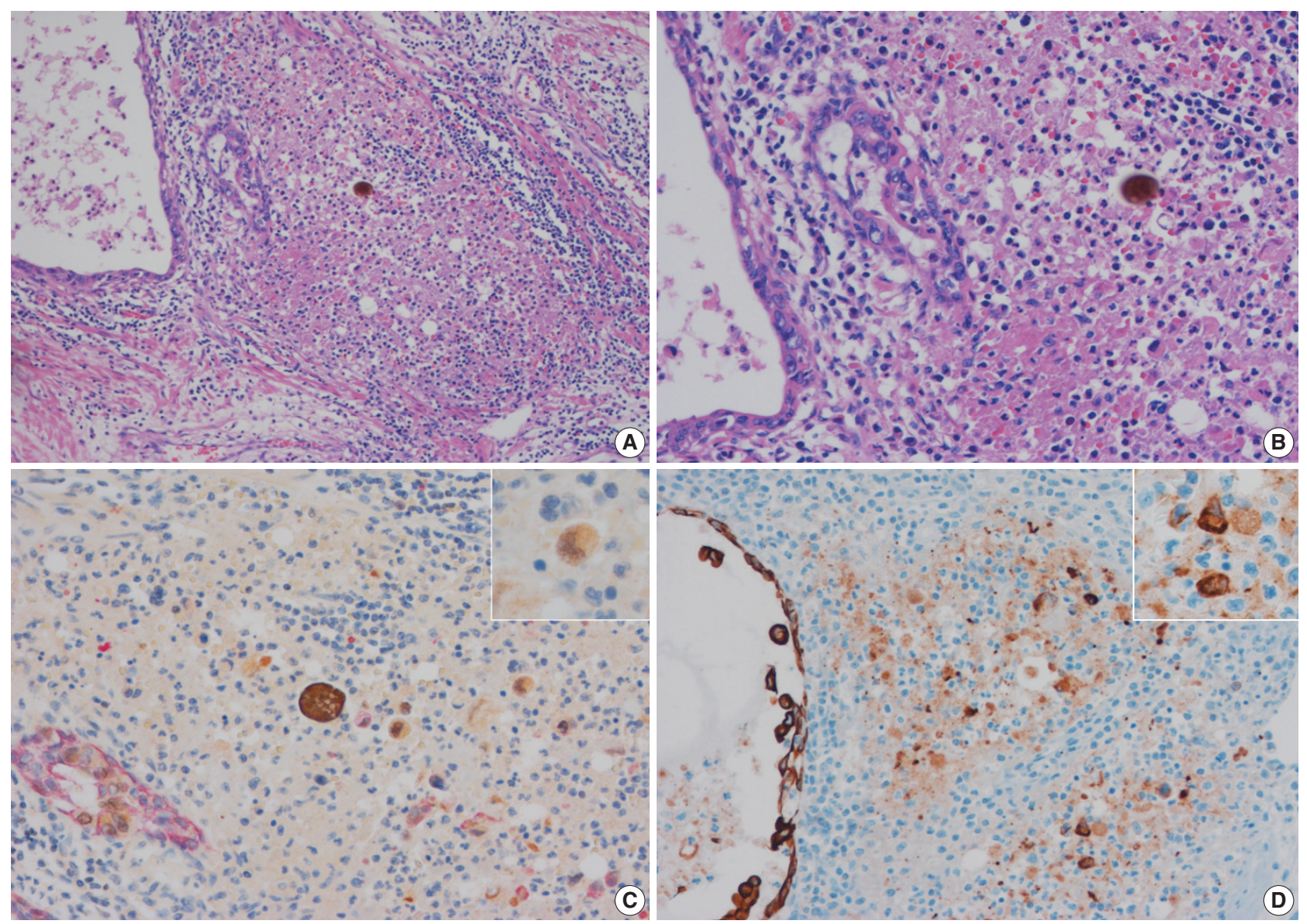

Fig. 3. (A) Periductal inflammatory infiltrates within prostatic stroma. (B) Inflammatory infiltrates with no discernible tumor cells. (C) GATA-3positive, high molecular weight cytokeratin-negative scattered tumor cells in inflammatory infiltrates (double immunohistochemical stain). (D) Scattered tumor cells are also positive for pancytokeratin stain.

nosis, but even a few cases with focal PSI may have similarly poor prognosis $[16,17]$. Therefore, it is essential to find even focal areas of PSI for proper classification. In 7th AJCC staging system, primary bladder UCs invading prostatic stroma through spreading to the prostatic urethra are classified as T4a. However, in the new 8th AJCC staging system [15], only those cases with primary bladder UCs directly invading prostatic stroma through the bladder wall are classified as T4a. Continuous subepithelial PSI from in situ UC is now categorized as T2 and does not affect the staging of primary bladder muscle-invasive UC (>T2). Yet distinguishing continuous subepithelial PSI of in situ UC (T2) from involvement of in situ UC (Tis) in non-muscle invasive primary bladder UCs (Tis or T1) are still important for patients who underwent early radical cystoprostatectomy because overall tumor stage can be upstaged because of the presence of PSI. Also, even though the impact of a concurrent continuous subepithelial PSI on the prognosis of muscle-invasive primary bladder UCs (> T2) is still unclear, the presence of PSI itself is reported to be associated with poor prognosis regardless of the mode of invasion [16].

Therefore, the significance of PSI needs to be re-evaluated. In most cases with PSI, stromal invasion can be easily detected on H\&E slides because PSI is usually associated with single isolate cell invasion, desmoplastic stromal reaction, acute and/or chronic inflammatory response, and retraction artifact around tumor cells [18]. However, the determination of PSI is somewhat subjective and may be impossible in some cases because they lack these characteristic histologic features on H\&E slides. Perrino et al. [19] showed $11.1 \%$ (4/36) discordant interpretations of PSI between original report and review using double immunostains with cytokeratin (CK) 7/CK5 and p53/CK5 [20]. The main distinction between in situ UC from PSI is to identify tumor cells in the prostatic stroma without surrounding prostatic basal cells. In this study, we evaluated the utility of HMWCK and GATA3 double IHC in determining stromal invasion of primary bladder UC in the prostate. We classified equivocal PSI cases into two 
patterns on $\mathrm{H} \& \mathrm{E}$. In all cases with pattern 1 invasion, the diagnoses based on $\mathrm{H} \& \mathrm{E}$ and on IHC were concordant. However, in cases showing pattern 2 , the concordance rate was low with two of three cases being misdiagnosed on H\&E. Singly scattered tumor cells were present in inflammatory stroma in those two cases. The singly scattered tumor cells were positive for pancytokeratin as well as GATA-3 but negative for HMWCK. Based on these findings, we recommend pancytokeratin and GATA-3 IHC stains in the prostate with pattern 2 equivocal PSI.

In previous two studies, various combinations of double stains were used to differentiate in situ UC from PSI in the prostate [18,20]. Chastain et al. [18] demonstrated that p63 and HMWCK double stains are useful in differentiating PSI. Even though this combination of double IHC is useful when p63-positive tumor cells are present in the prostatic stroma without surrounding HMWCK-positive basal cells, it has a limitation because not all tumor cells are positive for p63. In all cases, basal cells showed strong staining for both p63 and HMWCK, but only $50 \%$ and $41 \%$ of cases showed no or weak expression in tumor cells, respectively [18]. In the other study, Fichtenbaum et al. [20] reported that double stains with $\mathrm{CK} 7 / \mathrm{CK} 5$ and p53/CK5 discriminated in situ vs. invasive urothelial cancer in the prostate. These combinations of double stains are also a good method to discriminate in situ vs. PSI and can be utilized in a routine practice because $\mathrm{CK} 5$ is a very sensitive and specific marker for basal cells $(100 \%)$ and $\mathrm{CK} 7$ and p53 are very sensitive markers for tumor cells with $100 \%$ and $83 \%$ positivity, respectively [20]. $\mathrm{CK} 7$ is very specific for UCs in differentiating commonly encountered adenocarcinomas such as prostatic adenocarcinoma and metastatic colonic adenocarcinoma because the expression of CK7 is not usually seen in these adenocarcinomas $[21,22]$. However, even though rare, other metastatic adenocarcinomas including pulmonary adenocarcinoma, ovarian serous adenocarcinoma, endometrial adenocarcinoma, gastric adenocarcinoma, cholangiocarcinoma, invasive ductal carcinoma, that can be found in the prostate also may express CK7 [21,22]. Moreover, high grade UC may be negative for $\mathrm{CK} 7$ occasionally [23]. In our study, we used GATA-3 stain for UCs and HMWCK for prostatic basal cells. GATA-3 is a recently described immunohistochemical marker that is specific and sensitive for UCs and breast cancers [24,25]. When GATA-3-positive tumor cells are present in the prostatic stroma, PSI by UCs is highly suggested in the proper clinical context.

There are several limitations in this study. First, selective limited prostate sectioning technique was applied to this study. If whole-mount step sections of the prostate were selected, more cases with prostatic involvement might have been detected. Second, the number of subjects, especially the number of cases with equivocal PSI, was rather small. Third, GATA-3 also stains lymphocytes. GATA-3-positive atypical cells were present in the inflammatory stroma (case 6). Even though GATA-3-positive atypical cells in the inflammatory stroma were confirmed as epithelial cells by pancytokeratin staining, they might be keratin-positive debris such as disrupted normal epithelium or disrupted prostatic ducts or glands, especially in post-BCG status. However, BCG instillation was not performed in this case. Fourth, GATA-3 is recently reported to show aberrant diffuse nuclear staining in both luminal and basal cells of benign prostate glands with radiation atypia as well as in the basal cells of non-irradiated benign prostate glands [26]. UC and benign prostate glands can be distinguished based on their morphology, but certain variants of UCs, especially UC with glandular differentiation can cause a diagnostic problem. Fifth, even though GATA-3 is a novel immunohistochemical marker for UC, GATA-3 expression differed among UC variants [27]. Especially, GATA-3 is reported to be weakly or rarely expressed in sarcomatoid and small cell carcinoma variants of UC and squamous cell carcinomas [27]. Thus, GATA-3 immunostain may have only limited diagnostic value in such cases. Lastly, strong HMWCK positivity is reported in $50 \%$ to $91.7 \%$ of UCs $[18,28]$.

In the current study, weak GATA3 positivity in basal cells of benign prostatic glands was observed in two out of seven cases (28.6\%) while HMWCK positivity in UCs was found in five out of seven (71.4\%). Such expression pattern could cause diagnostic confusion for pathologists in irradiated specimens which often accompany radiation atypia in benign prostatic glands. However, none of the patients in the current study had received previous irradiation therapy. Also, even though some benign prostatic glands expressed GATA3 positivity, the staining intensity was much weaker than that observed in UCs. Furthermore, none of GATA3 positive benign prostatic cells in the current study showed cytologic atypia.

The double staining for GATA-3 and HMWCK may enable us to distinguish PSI from equivocal in situ lesions. In rare cases in which GATA-3 is aberrantly expressed in prostatic glands, the presence of HMWCK staining and the architectural and cytological findings on $\mathrm{H} \& \mathrm{E}$ can aid in the correct interpretation. Another advantage of the double staining method is convenience. Compared to switching between GATA-3 and HMWCK stains individually to spot the equivocal lesion under microscopic examination, assessing a single slide is more convenient. Lastly, the equivocal PSI lesions tend to be subtle and minute in size. 
Double immunostain method reduces the possible loss of the lesion during multiple sections for different markers.

Differentiating cases of UC with PSI from in situ UC involving prostatic ducts or acini is critical for subsequent patients' prognosis even though the significance of focal PSI is still controversial and PSI itself is not considered as pT4a any longer in 8th AJCC staging system. However, one patient with PSI (case 6, original T3 [7th] $\rightarrow$ revised to T4a [7th], T3 [8th]) died with only 16 months follow-up after cystoprostatectomy. On the contrary, the other patient without PSI (case 5, original T4a [7th] $\rightarrow$ revised to T1 [7th], T1 [8th]) is still alive for 54 months. In these equivocal cases, one case (case 5) was misdiagnosed as PSI and subsequently T4a (7th AJCC) was assigned in the original diagnosis. The presence of basal cells around tumor nests, as demonstrated by double IHC, led to the final diagnosis of in situ UC involving prostatic ducts in this case. The other case (case 6) was diagnosed as in situ involving only prostatic ducts without PSI in the original diagnosis; therefore, pT3 was based on the perivesical fat invasion of the primary bladder UC. However, PSI besides in situ prostatic duct involvement was diagnosed because scattered infiltrating tumor cells with positive GATA3 and pancytokeratin positivity with lack of HMWCK stain within the inflammatory infiltrates and subsequently pT4a was re-assigned in this case. Another patient with PSI (case 7, original T4a [7th] $\rightarrow$ revised to T4a [7th], T2 [8th]) is also alive for 58 months. In this case, the primary bladder UC was nonmuscle invasive and the concurrent urethral PSI determined the staging.

In conclusion, we demonstrated that GATA-3 and HMWCK double IHC is useful in discriminating PSI by UC from in situ UC involving prostatic ducts or acini especially in cases with pattern 2 equivocal PSI.

\section{ORCID}

Junghye Lee: https://orcid.org/0000-0001-8454-4360

Youngeun Yoo: https://orcid.org/0000-0003-4855-3870

Sanghui Park: https://orcid.org/0000-0003-3837-8677

Min-Sun Cho: https://orcid.org/0000-0001-8772-9686

Sun Hee Sung: https://orcid.org/0000-0001-9345-1131

Jae Y. Ro: https://orcid.org/0000-0002-4158-3658

\section{Author Contributions}

Conceptualization: SP, JYR.

Data curation: SP, YY, JL.

Formal analysis: JL, SP.
Investigation: JL, SP.

Methodology: SP.

Project administration: SP.

Resources: SP, SHS, MSC.

Supervision: SP.

Validation: SP, SHS, MSC.

Visualization: JL, SP.

Writing—original draft: JL, SP.

Writing—review \& editing: JL, SP.

\section{Conflicts of Interest}

The authors declare that they have no potential conflicts of interest.

\section{Funding}

No funding to declare.

\section{REFERENCES}

1. Bates HR Jr. Transitional cell carcinoma of the prostate. J Urol 1969; 101: 206-7.

2. Rubenstein AB, Rubnitz ME. Transitional cell carcinoma of the prostate. Cancer 1969; 24: 543-6.

3. Wolfe JH, Lloyd-Davies RW. The management of transitional cell carcinoma in the prostate. Br J Urol 1981; 53: 253-7.

4. Schellhammer PF, Bean MA, Whitmore WF Jr. Prostatic involvement by transitional cell carcinoma: pathogenesis patterns and prognosis. J Urol 1977; 118: 399-403.

5. Pettus JA, Al-Ahmadie H, Barocas DA, et al. Risk assessment of prostatic pathology in patients undergoing radical cystoprostatectomy. Eur Urol 2008; 53: 370-5.

6. Reese JH, Freiha FS, Gelb AB, Lum BL, Torti FM. Transitional cell carcinoma of the prostate in patients undergoing radical cystoprostatectomy. J Urol 1992; 147: 92-5.

7. Revelo MP, Cookson MS, Chang SS, Shook MF, Smith JA Jr, Shappell SB. Incidence and location of prostate and urothelial carcinoma in prostates from cystoprostatectomies: implications for possible apical sparing surgery. J Urol 2004; 171(2 Pt 1): 646-51.

8. Shen SS, Lerner SP, Muezzinoglu B, Truong LD, Amiel G, Wheeler TM. Prostatic involvement by transitional cell carcinoma in patients with bladder cancer and its prognostic significance. Hum Pathol 2006; 37: 726-34.

9. Wood DP Jr, Montie JE, Pontes JE, VanderBrug Medendorp S, Levin HS. Transitional cell carcinoma of the prostate in cystoprostatectomy specimens removed for bladder cancer. J Urol 1989; 141: 346-9. 
10. Coutts AG, Grigor KM, Fowler JW. Urethral dysplasia and bladder cancer in cystectomy specimens. Br J Urol 1985; 57: 535-41.

11. Herr HW, Donat SM. Prostatic tumor relapse in patients with superficial bladder tumors: 15-year outcome. J Urol 1999; 161: 1854-7.

12. Njinou Ngninkeu B, Lorge F, Moulin P, Jamart J, Van Cangh PJ. Transitional cell carcinoma involving the prostate: a clinicopathological retrospective study of 76 cases. J Urol 2003; 169: 149-52.

13. Pagano F, Bassi P, Ferrante GL, et al. Is stage pT4a (D1) reliable in assessing transitional cell carcinoma involvement of the prostate in patients with a concurrent bladder cancer? A necessary distinction for contiguous or noncontiguous involvement. J Urol 1996; 155: 244-7.

14. Edge SB, Byrd DR, Compton CC, et al. AJCC cancer staging manual. 7th ed. New York: Springer, 2010.

15. Amin MB, Edge S, Greene F, et al. AJCC cancer staging manual. Chicago: Springer, 2017.

16. Ayyathurai R, Gomez P, Luongo T, Soloway MS, Manoharan M. Prostatic involvement by urothelial carcinoma of the bladder: clinicopathological features and outcome after radical cystectomy. BJU Int 2007; 100: 1021-5.

17. Oliva IV, Smith SL, Chen Z, Osunkoya AO. Urothelial carcinoma of the bladder with transmural and direct prostatic stromal invasion: does extent of stromal invasion significantly impact patient outcome? Hum Pathol 2011; 42: 51-6.

18. Chastain EC, Oliva IV, Osunkoya AO. Utility of p63 and high molecular weight cytokeratin in the distinction between urothelial carcinoma with prostatic stromal invasion and urothelial carcinoma with colonisation of prostatic ducts and acini. Pathology 2012; 44: 199-203.

19. Perrino CM, Fichtenbaum EJ, Pohar KS, Zynger DL. Challenges in the pathological reporting of urothelial carcinoma involving prostatic transurethral resection specimens within a single institution. Pathology 2013; 45: 664-9.
20. Fichtenbaum EJ, Marsh WL Jr, Zynger DL. CK5, CK5/6, and double-stains CK7/CK5 and p53/CK5 discriminate in situ vs invasive urothelial cancer in the prostate. Am J Clin Pathol 2012; 138: 190-7.

21. Kim DH, Joo JE, Kim EK, Lee HJ, Lee WM. The Expressions of Cytokeratin 7 and 20 in Epithelial Tumors: A Survey of 91 Cases. Cancer Res Treat 2003; 35: 355-63.

22. Seipel AH, Samaratunga H, Delahunt B, Wiklund P, Clements M, Egevad L. Immunohistochemistry of ductal adenocarcinoma of the prostate and adenocarcinomas of non-prostatic origin: a comparative study. APMIS 2016; 124: 263-70.

23. Downes MR, Torlakovic EE, Aldaoud N, Zlotta AR, Evans AJ, van der Kwast TH. Diagnostic utility of androgen receptor expression in discriminating poorly differentiated urothelial and prostate carcinoma. J Clin Pathol 2013; 66: 779-86.

24. Higgins JP, Kaygusuz G, Wang L, et al. Placental S100 (S100P) and GATA3: markers for transitional epithelium and urothelial carcinoma discovered by complementary DNA microarray. Am J Surg Pathol 2007; 31: 673-80.

25. Liu H, Shi J, Wilkerson ML, Lin F. Immunohistochemical evaluation of GATA3 expression in tumors and normal tissues: a useful immunomarker for breast and urothelial carcinomas. Am J Clin Pathol 2012; 138: 57-64.

26. Tian W, Dorn D, Wei S, et al. GATA3 expression in benign prostate glands with radiation atypia: a diagnostic pitfall. Histopathology 2017; 71: 150-5.

27. Liang Y, Heitzman J, Kamat AM, Dinney CP, Czerniak B, Guo CC. Differential expression of GATA-3 in urothelial carcinoma variants. Hum Pathol 2014; 45: 1466-72.

28. Chuang AY, DeMarzo AM, Veltri RW, Sharma RB, Bieberich CJ, Epstein JI. Immunohistochemical differentiation of high-grade prostate carcinoma from urothelial carcinoma. Am J Surg Pathol 2007; 31: 1246-55. 